\title{
Patient Characteristics and Outcomes of Relapsed/ Refractory Multiple Myeloma in Patients Treated with Proteasome Inhibitors in Germany
}

\author{
H. Tilman Steinmetz ${ }^{a}$ Moushmi Singh ${ }^{b} \quad$ Andrea Lebioda $^{c}$ \\ Sebastian Gonzalez-McQuire $^{d}$ Achim Rieth $^{e}$ Martina Schoehl $^{\mathrm{e}}$ \\ Wolfram Poenisch ${ }^{f}$ \\ ${ }^{\mathrm{a} O n c o l o g y}$ Cologne, Center for Hematology and Oncology, Cologne, Germany; ${ }^{\mathrm{b} H e a l t h ~ E c o n o m i c s, ~ A m g e n ~ L t d ., ~}$ \\ Uxbridge, UK; ${ }^{C}$ Health Economics, Amgen GmbH, Munich, Germany; ${ }^{d}$ Health Economics, Amgen (Europe) GmbH, \\ Rotkreuz, Switzerland; ${ }^{e}$ Medical Development, Amgen GmbH, Munich, Germany; ${ }^{f}$ Department of Hematology and \\ Cellular Therapy, Medical Clinic and Policlinic I, University of Leipzig, Leipzig, Germany
}

\section{Keywords}

Multiple myeloma $\cdot$ Chart review $\cdot$ Proteasome inhibitors

\begin{abstract}
Introduction: Real-world data reflects treatments and outcomes in clinical practice in contrast with controlled clinical trials. This study evaluates real-life multiple myeloma (MM) patients receiving proteasome inhibitor (PI)-based treatments in the second or third therapy line in 2017 in Germany. Methods: This is a retrospective chart review on adult relapsed/refractory MM patients treated with $\geq 1$ dose of a PIbased regimen in either the second or the third line of therapy. Participating physicians had $\geq 3$ years of clinical experience in treating symptomatic MM patients and used $\mathrm{PI}$ according to the label. Results: Distinct patient profiles for each PI-based regimen emerged. Younger, fitter, transplanteligible patients received novel PI triplets such as carfilzomib in combination with lenalidomide and dexamethasone (KRd) or IRd. Patients receiving lenalidomide in first-line therapy mostly received lenalidomide-free regimens in secondline therapy. In high-risk patients, no clear treatment patterns could be ascertained. The complete response rates were highest with $\mathrm{KRd}(13.0 \%)$, followed by carfilzomib in combination with dexamethasone (Kd) (5.7\%) and bortezomib (4.8\%). The very good partial response rates were highest with IRd (76.9\%), followed by KRd (53.7\%), Kd (25.7\%), and bortezomib (20.5\%). None of the KRd- or IRd-treated pa-
\end{abstract}

karger@karger.com www.karger.com/ort

Karger ${ }^{\prime \prime}$

马OPEN ACCESS
(C) 2020 The Author(s)

Published by S. Karger AG, Basel

This is an Open Access article licensed under the Creative Commons Attribution-NonCommercial-4.0 International License (CC BY-NC) (http://www.karger.com/Services/OpenAccessLicense), applicable to nd distribution for com- tients responded below a partial response. Discussion/Conclusion: Clear patient profiles for each PI type were observed. In second-line therapy, younger, fitter, transplant-eligible patients received novel-PI-based triplets, e.g., KRd or IRd. Patients treated with lenalidomide in first-line therapy mostly received lenalidomide-sparing regimens in second-line therapy. In high-risk patients no clear treatment patterns could be ascertained due to the limited sample size.

(c) 2020 The Author(s)

Published by S. Karger AG, Basel

\section{Introduction}

Multiple myeloma (MM) is a neoplastic disorder characterized by a monoclonal proliferation of plasma cells in the bone marrow. Symptoms include hypercalcemia, renal dysfunction, symptoms secondary to the suppression of normal hematopoiesis such as anemia, skeletal destruction of the bones that results in bone pain and fractures, and a high concentration of immunoglobulin and secondary immunodeficiency. MM is the second most common hematological malignancy ( $10 \%$ of all blood cancers, after non-Hodgkin lymphoma) and constitutes $1 \%$ of all cancers [1]. In Germany, 6,500 patients were newly diagnosed with MM in 2014, and approximately 4,000 deaths were attributed to MM in the same year [2,3].

The 5-year survival of MM patients is $49.6 \%$ according to the National Cancer Institute's Surveillance, Epidemi- 
ology, and End Results Program (SEER) [4]. However, there is a great variability among patients and survival can be up to 20 years. In Germany, the relative overall 5-year survival rate is approximately $50 \%[2,3]$.

In Europe, the age-standardized incidence rate per 100,000 persons was estimated at 4.7 for males and 3.1 for females [5]. In Germany, the age-standardized incidence rate was estimated at 5.4-6.0 for males and 3.5-3.9 for females, depending on the source $[5,6]$. The incidence rate steadily increases with age, with a peak age-specific incidence rate of approximately $40-50$ per 100,000 for males and approximately $25-30$ for females over 75 years of age [6]. As the general population ages, MM incidence rates are expected to increase accordingly [7].

Diagnosis of MM depends on the identification of monoclonal plasma cells in the bone marrow, M-protein in the serum and/or urine, osteolytic lesions, and an indicative clinical profile (fatigue, weakness, and loss of appetite and weight) $[7,8]$.

Treatment of MM commonly starts when patients become symptomatic. Newly diagnosed asymptomatic patients are watched until symptoms develop, unless they are identified as having active disease according to the International Myeloma Working Group (IMWG) definition [9]. Initial treatment decisions are based on whether stem cell transplantation (SCT) will be a part of the strategy, and they mainly depend on the patient's age and comorbidities. Depending on the patient's clinical condition and eligibility for an autologous stem cell transplant, an autologous stem cell transplant is used after induction and followed by maintenance therapies [10]. For transplant-ineligible patients, several options are recommended for first-line treatment, including bortezomib- and lenalidomide-based regimens [11].

Although it is now possible to achieve a long remission, the natural evolution of the disease leads to relapse after upfront treatment [12]. In recent years, multiple new drugs have been introduced for the treatment of MM in the relapsed setting. In 2004, bortezomib was the first proteasome inhibitor (PI) to be approved by the European Medicines Agency (EMA). PI have been proven to be an effective strategy in MM treatment. More than 10 years after the introduction of bortezomib, 2 novel PI were approved in Europe, i.e., carfilzomib, in combination with lenalidomide and dexamethasone (November 2015) and in combination with dexamethasone alone (July 2016), and ixazomib in combination with lenalidomide and dexamethasone (November 2016).

There is only scarce data on patient profiles, treatment combinations and sequences, and associated efficacy profiles in relapsed and/or refractory MM in the real-world setting; additionally, studies of treatment landscapes underlie fast-moving developments in clinical practice [1316]. Importantly, recent publications have not focused on
PI. Therefore, it was deemed important to identify the characteristics of patients receiving PI and their prior treatment pathways in a real-world setting in Germany in $2017 / 2018$.

\section{Materials and Methods}

\section{Study Objectives}

The primary objectives of this study were to describe the demographic and clinical characteristics of patients treated with a PI (bortezomib, carfilzomib, or ixazomib) at diagnosis and at PI initiation of the respective line of observation. Antimyeloma treatment history by PI regimen and PI-based treatment patterns were analyzed. The secondary objective was to describe the best response achieved with a PI-based treatment. The best response was defined as a complete response (CR) or a very good partial response (VGPR). The response was assessed by the treating physicians and not necessarily based on conventionally defined criteria [13]. CR assessment was performed either by bone marrow aspiration or by the physicians based on their usual practice. For VGPR or less, a response assessment based on $\mathrm{M}$ protein was enough.

\section{Study Design}

This national retrospective medical chart review included consecutive patients treated with at least 1 dose of a PI-based regimen in participating hospitals/centers across Germany between January and June 2017. The data collection ended in April 2018. Physicians completed paper case report forms (CRF) from the medical charts of eligible patients, and data from all available treatment lines prior to or following the index PI was extracted. The study used 1 CRF per treatment line of interest and both sets were analyzed separately. After completion of the CRF, patient inclusion criteria were verified and the priority data in the CRF was validated by external epidemiology research assistants in the presence of the investigating physician.

\section{Eligibility Criteria}

Physicians were eligible if they had at least 3 years of clinical experience, had been personally responsible for managing and treating symptomatic MM patients, and had been using PI in daily practice according to their respective approved indications. Each physician was required to provide at least 4 medical records.

Selection criteria for patient records were: diagnosis of MM, age $\geq 18$ years at the time of diagnosis, relapsed or refractory status from at least 1 prior line of therapy, and at least 1 dose of PI in second- or third-line therapy between January 1 and June 30, 2017, irrespectively of when the PI treatment was started. Patients with MGUS or smoldering myeloma or who received a PI in the relapsed setting within a clinical trial were not eligible.

\section{Statistical Analysis}

Descriptive statistics were used. Numerical variables are shown as means $( \pm \mathrm{SD})$ or medians ( $95 \% \mathrm{CI}$ or first and third quartile). Categorical/ordinal variables are shown as numbers (\%) of patients who fall into each response category. For exploratory timeto-event variables, i.e., duration of treatment, time to next treatment, progression-free survival (PFS), and observed survival (time of death), Kaplan-Meier plots and quartiles, including the median time to event, were provided. The time to next treatment was defined as the time from treatment initiation (of the analyzed line) to initiation of the next line of therapy, and duration of treatment was defined as the time from PI initiation to the end of PI treatment or death, whichever occurred first. CRF were completed for patients 
Table 1. Characteristics of patients receiving a PI in the second line of treatment

\begin{tabular}{|c|c|c|c|c|c|c|}
\hline Variable & $\begin{array}{l}\text { Total 2L } \\
(n=219)\end{array}$ & $\begin{array}{l}2 \mathrm{~L} \mathrm{BTZ} \\
(n=92)\end{array}$ & $\begin{array}{l}\text { 2L CFZ } \\
(n=106)\end{array}$ & $\begin{array}{l}2 \mathrm{~L} \text { KRd } \\
(n=59)\end{array}$ & $\begin{array}{l}2 \mathrm{~L} \mathrm{Kd} \\
(n=47)\end{array}$ & $\begin{array}{l}\text { 2L IXA } \\
(n=21)\end{array}$ \\
\hline \multicolumn{7}{|l|}{ Gender } \\
\hline Male & $152(69.4)$ & $65(70.7)$ & $71(67.0)$ & $40(67.8)$ & $31(66.0)$ & $16(76.2)$ \\
\hline Female & $67(30.6)$ & $27(29.3)$ & $35(33.0)$ & $19(32.2)$ & $16(34.0)$ & $5(23.8)$ \\
\hline Age at diagnosis, years & $69(24-85)$ & $72(55-85)$ & $64.5(24-82)$ & $62(41-80)$ & $69(24-82)$ & $65(47-83)$ \\
\hline \multicolumn{7}{|c|}{ Age category at diagnosis (years) } \\
\hline$<65$ & $73(33.3)$ & $10(10.9)$ & $53(50.0)$ & $40(67.8)$ & $13(27.7)$ & $10(47.6)$ \\
\hline $65-74$ & $92(42.0)$ & $48(52.2)$ & $38(35.8)$ & $16(27.1)$ & $22(46.8)$ & $6(28.6)$ \\
\hline$\geq 75$ & $54(24.7)$ & $34(37.0)$ & $15(14.2)$ & $3(5.1)$ & $12(25.5)$ & $5(23.8)$ \\
\hline Age at initiation of $2 \mathrm{~L}$ & $70(26-89)$ & $74(59-88)$ & $68(26-83)$ & $65(42-81)$ & $71(26-83)$ & $66(47-89)$ \\
\hline \multicolumn{7}{|c|}{ Age category at initiation of $2 \mathrm{~L}$ (years) } \\
\hline$<65$ & $52(23.7)$ & $6(6.5)$ & $38(35.8)$ & $28(47.5)$ & $10(21.3)$ & $8(38.1)$ \\
\hline $65-74$ & $93(42.5)$ & $44(47.8)$ & $42(39.6)$ & $22(37.3)$ & $20(42.6)$ & $7(33.3)$ \\
\hline$\geq 75$ & $74(33.8)$ & $42(45.7)$ & $26(24.5)$ & $9(15.3)$ & $17(36.2)$ & $6(28.6)$ \\
\hline \multicolumn{7}{|l|}{ ECOG at diagnosis } \\
\hline $0-1$ & $119(54.3)$ & $35(38.0)$ & $68(64.2)$ & $52(88.1)$ & $16(34.0)$ & $16(76.2)$ \\
\hline 2 & $98(44.7)$ & $56(60.9)$ & $37(34.9)$ & $7(11.9)$ & $30(63.8)$ & $5(23.8)$ \\
\hline $3-4$ & $2(0.9)$ & $1(1.1)$ & $1(0.9)$ & $0(0.0)$ & $1(2.1)$ & $0(0.0)$ \\
\hline \multicolumn{7}{|l|}{ ECOG at initiation of $2 \mathrm{~L}$} \\
\hline $0-1$ & $118(53.9)$ & $39(42.4)$ & $64(60.4)$ & $53(89.8)$ & $11(23.4)$ & $15(71.4)$ \\
\hline 2 & $100(45.7)$ & $52(56.5)$ & $42(39.6)$ & $6(10.2)$ & $36(76.6)$ & $6(28.6)$ \\
\hline $3-4$ & $1(0.5)$ & $1(1.1)$ & $0(0.0)$ & $0(0.0)$ & $0(0.0)$ & $0(0.0)$ \\
\hline \multicolumn{7}{|l|}{ Work status } \\
\hline Employed/on job & $43(19.6)$ & $4(4.3)$ & $32(30.2)$ & $25(42.4)$ & $7(14.9)$ & $7(33.3)$ \\
\hline None & $176(80.4)$ & $88(95.7)$ & $74(69.8)$ & $34(57.6)$ & $40(85.1)$ & $14(66.7)$ \\
\hline
\end{tabular}

Values are presented as numbers (\%) or medians (range). 2L, second line of treatment; BTZ, bortezomib; CFZ, carfilzomib; IXA, ixazomib.

with at least 1 dose of a PI in either second- or third-line therapy; data specific to each line was separately analyzed.

No statistical modeling was applied to the study results and no sensitivity analyses were performed on this dataset. Due to the retrospective nature of this study, missing and/or incomplete data was expected in some of the medical records. All missing values were documented and excluded from the tables when necessary. No patients were excluded from the overall data set; however, some patients were excluded from specific analyses if they had a missing value for that specific variable.

\section{Results}

Overall, 44 investigators, all onco-hematologists, participated in this study. Of these, $56.8 \%(n=25)$ were office based, $43.2 \%(n=19)$ were hospital based, $20.5 \%(n=9)$ worked in a transplant center, $22.7 \%(n=10)$ worked in a hospital that was formally part of a network of transplant centers, and $56.8 \%(n=25)$ were not formally part of a transplant network. These 44 investigators documented a total of 302 patients. Of these, $72.5 \%(n=219)$ were documented as having received a PI in second-line and $27.5 \%(n=83)$ in third-line therapy.

\section{Patients Receiving a PI in Second-Line Therapy}

Patient Characteristics

Of the 219 patients receiving PI in second-line therapy, most were male $(69.4 \%, n=152)$; they had a median age of 69 years (range 24-85) at diagnosis and 70 years (range 26-89) at the initiation of second-line treatment. There were some differences between the different PI groups with regard to age, with patients receiving carfilzomib- or ixazomib-based triplet regimens being younger. Approximately $30 \%$ of the patients receiving novel PI were still employed or on their job compared to $4.3 \%(n=4)$ of those receiving a bortezomib-based regimen (Table 1 ).

\section{Disease Characteristics}

The common myeloma symptoms suggesting a diagnosis of myeloma (circumstances at diagnosis) were: bone pain in $58.9 \%(n=129)$, anemia in $35.6 \%(n=78)$, hypercalcemia in $18.7 \%(n=41)$, and renal dysfunction in $8.2 \%$ ( $n=18$; online suppl. Table S1; see www.karger. com/doi/10.1159/000509018 for all online suppl. material) of patients. A history of skeletal related events (SRE; defined as pathological fractures, spinal cord compression, necessity for radiation or surgery to the bone) was 
Table 2. Disease characteristics of patients receiving a PI in the second line of treatment

\begin{tabular}{lcccccc}
\hline Variable & $\begin{array}{c}\text { Total 2L } \\
(n=219)\end{array}$ & $\begin{array}{l}\text { 2L BTZ } \\
(n=92)\end{array}$ & $\begin{array}{l}\text { 2L CFZ } \\
(n=106)\end{array}$ & $\begin{array}{l}\text { 2L KRd } \\
(n=59)\end{array}$ & $\begin{array}{l}\text { 2L Kd } \\
(n=47)\end{array}$ & $\begin{array}{l}\text { 2L IXA } \\
(n=21)\end{array}$ \\
\hline ISS at diagnosis & & & & & \\
ISS score available & $210(95.9)$ & $87(94.6)$ & $102(96.2)$ & $55(93.2)$ & $47(100.0$ & $21(100.0)$ \\
I & $15(7.1)$ & $4(4.6)$ & $9(8.8)$ & $8(14.5)$ & $1(2.1)$ & $2(9.5)$ \\
II & $101(48.1)$ & $35(40.2)$ & $58(56.9)$ & $25(45.5)$ & $33(70.2)$ & $8(38.1)$ \\
III & $94(44.8)$ & $48(55.2)$ & $35(34.3)$ & $22(40.0)$ & $13(27.7)$ & $11(52.4)$ \\
\hline Cytogenetic abnormalities at diagnosis & & & & & \\
Yes (at least 1 tested) & $105(47.9)$ & $26(28.3)$ & $65(61.3)$ & $47(79.7)$ & $18(38.3)$ & $14(66.7)$ \\
del(17p13) & $98(44.7)$ & $22(23.9)$ & $62(58.5)$ & $47(79.7)$ & $15(31.9)$ & $14(66.7)$ \\
t(4;14) & $91(41.6)$ & $21(22.8)$ & $59(55.7)$ & $46(78.0)$ & $13(27.7)$ & $11(52.4)$ \\
t(14;16) & $94(42.9)$ & $21(22.8)$ & $59(55.7)$ & $46(78.0)$ & $13(27.7)$ & $14(66.7)$ \\
del(13) & $103(47.0)$ & $25(27.2)$ & $64(60.4)$ & $47(79.7)$ & $17(36.2)$ & $14(100.0)$ \\
amp(1q21) & $89(40.6)$ & $21(22.8)$ & $58(54.7)$ & $46(78.0)$ & $12(25.5)$ & $10(47.6)$ \\
t(11;14) & $95(43.4)$ & $24(26.1)$ & $60(56.6)$ & $46(78.0)$ & $14(29.8)$ & $11(52.4)$ \\
None tested & $114(52.1)$ & $66(71.7)$ & $41(38.7)$ & $12(20.3)$ & $29(61.7)$ & $7(33.3)$ \\
Aberration detected & $105(47.9)$ & $26(28.3)$ & $65(61.3)$ & $47(79.7)$ & $18(38.3)$ & $14(66.7)$ \\
del(17p13) & $11(11.2)$ & $2(9.1)$ & $8(12.9)$ & $5(10.6)$ & $3(20.0)$ & $1(7.1)$ \\
t(4;14) & $15(16.5)$ & $5(23.8)$ & $7(11.9)$ & $5(10.6)$ & $2(15.4)$ & $3(27.3)$ \\
t(14;16) & $5(5.3)$ & $1(4.8)$ & $4(6.8)$ & $2(4.3)$ & $2(15.4)$ & $0(0.0)$ \\
del(13) & $23(22.3)$ & $9(36.0)$ & $13(20.3)$ & $9(19.1)$ & $4(23.5)$ & $1(7.1)$ \\
amp(1q21) & $14(15.7)$ & $0(0.0)$ & $14(24.1)$ & $14(30.4)$ & $0(0.0)$ & $0(0.0)$ \\
t(11;14) & $4(4.2)$ & $2(8.3)$ & $2(3.3)$ & $1(2.2)$ & $1(7.1)$ & $0(0.0)$ \\
\hline
\end{tabular}

Values are presented as numbers (\%). 2L, second line of treatment; BTZ, bortezomib; CFZ, carfilzomib; ISS; international staging system; IXA, ixazomib.

Fig. 1. PI-based regimens received in second-line therapy $(n=219)$. DVd, daratumumab in combination with bortezomib and dexamethasone; Id, ixazomib in combination with dexamethasone; VCd, bortezomib in combination with cyclophosphamide and dexamethasone.

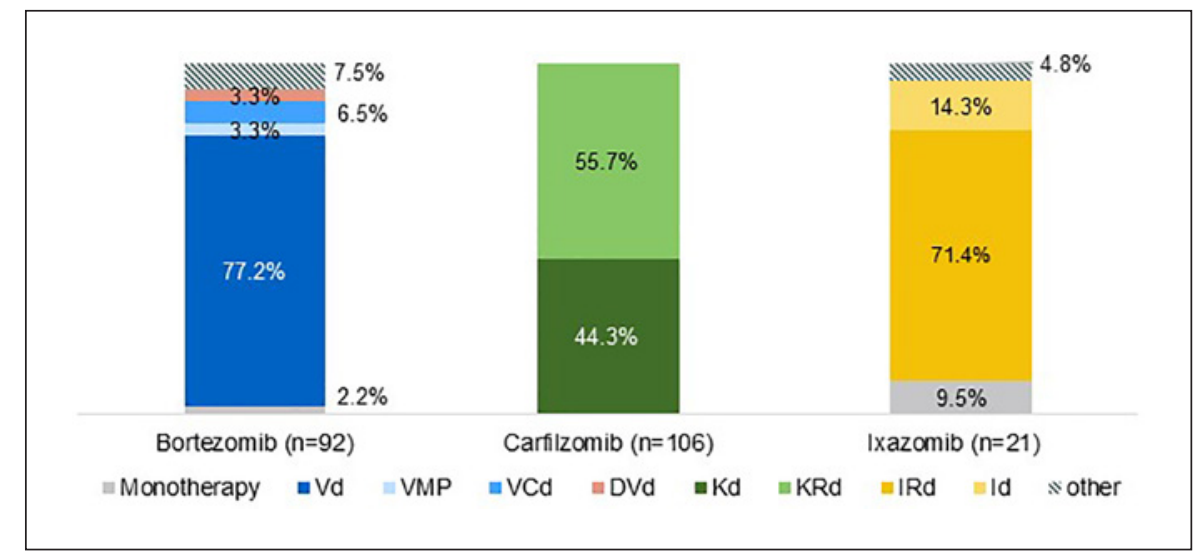

present in $34.2 \%(n=75)$ of the patients. The median (Q1, Q3) number of bone lesions at diagnosis was $1.0(0.0$, $35.0)$ and $2.0(0.0,50.0)$ at the initiation of second-line therapy (online suppl. Table S2). Renal function at diagnosis was normal in $60.7 \%(n=133)$ of the patients and at the initiation of second-line therapy it was normal in $53.4 \%$ ( $n=117$; online suppl. Table S3). At diagnosis, a history of deep vein thrombosis was documented in $4.6 \%$ $(n=10)$ of the patients, a history of neuropathy was found in $18.7 \%(n=41)$ of the patients, and a history of significant cardiovascular disease was seen in $16.9 \%(n=37$; online suppl. Table S4) of the patients. The described disease characteristics varied to some extent between prescribed regimens (online suppl. Tables S1 to S4). ISS stage and cytogenetic disposition also varied across PI (Table 2).

Treatments Received in Second-Line Therapy

As a second-line PI treatment, $42.0 \%(n=92)$ of the patients received a bortezomib-based regimen, $48.4 \%$ $(n=106)$ received a carfilzomib-based regimen, and 9.6\% $(n=21)$ received an ixazomib-based regimen. Figure 1 
Table 3. Transplant history of patients receiving a PI in the second line of treatment

\begin{tabular}{lcccccc}
\hline Variable & $\begin{array}{l}\text { Total 2L } \\
(n=219)\end{array}$ & $\begin{array}{l}\text { 2L BTZ } \\
(n=92)\end{array}$ & $\begin{array}{l}\text { 2L CFZ } \\
(n=106)\end{array}$ & $\begin{array}{l}\text { 2L KRd } \\
(n=59)\end{array}$ & $\begin{array}{l}\text { 2L Kd } \\
(n=47)\end{array}$ & $\begin{array}{l}\text { 2L IXA } \\
(n=21)\end{array}$ \\
\hline Transplant history & & & & & & \\
At least 1 transplantation & $72(32.9)$ & $13(14.1)$ & $52(49.1)$ & $43(72.9)$ & $9(19.1)$ & $7(33.3)$ \\
$\quad$ Transplant in 1L only & $65(29.7)$ & $9(9.8)$ & $49(46.2)$ & $40(67.8)$ & $9(19.1)$ & $7(33.3)$ \\
$\quad$ Transplant in 1L and 2L & $6(8.3)$ & $4(4.3)$ & $2(1.9)$ & $2(3.4)$ & $0(0.0)$ & $0(0.0)$ \\
$\quad$ Transplant in 2L only & $1(0.5)$ & $0(0.0)$ & $1(0.9)$ & $1(1.7)$ & $0(0.0)$ & $0(0.0)$ \\
No transplant & $147(67.1)$ & $79(85.9)$ & $54(50.9)$ & $16(27.1)$ & $38(80.9)$ & $14(66.7)$ \\
\hline
\end{tabular}

Values are presented as numbers (\%). 1L, first line of treatment; 2L, second line of treatment; BTZ, bortezomib; CFZ, carfilzomib; ISS; international staging system; IXA, ixazomib.

shows the distribution of the individual regimens prescribed for each PI type. Of the patients receiving bortezomib-based regimens, most received a doublet, mainly bortezomib-dexamethasone $(\mathrm{Vd})$. Of the carfilzomibbased regimens, slightly more than half of the patients received carfilzomib-lenalidomide-dexamethasone (KRd), and of ixazomib-based regimens, most patients received ixazomib-lenalidomide-dexamethasone (IRd).

Treatment History Prior to Second-Line Therapy

Of the patients receiving bortezomib-based regimens in second-line therapy $(n=92)$, the majority $(62.0 \%, n=$ 57) had been prescribed a lenalidomide-based regimen in first-line therapy. Of the patients receiving a carfilzomibbased regimen $(n=106), 78.3 \%(n=83)$ had received a prior bortezomib-based regimen. Of the patients receiving carfilzomib in combination with dexamethasone (Kd) $(n=47), 34.0 \%(n=16)$ had received prior lenalidomide versus none of the patients receiving KRd $(n=59)$. Patients receiving ixazomib in second-line therapy also mostly had received prior bortezomib $(66.7 \%, n=14)$. Figure 2 shows a Sankey diagram of treatment shifts from first to second line for all of the patients, and online supplementary Figures S1A and S1B show the same for patients with and without SCT. The median (Q1, Q3) duration between diagnosis and initiation of second-line therapy was 17.0 months $(10.0,33.0)$ overall, with variations between individual PI groups (online suppl. Table S5). The time from first- to second-line treatment was 16.0 months overall, with wide interindividual ranges and trends showing intergroup differences (online suppl. Table S6). Supportive treatments received in the second line are listed in online supplementary Table S11. Notably, most patients $(88.1 \%, n=193)$ received a concomitant osteoprotective therapy.

Transplant History

Overall, 32.9\% $(n=72)$ of second-line PI patients had received at least 1 transplantation, mostly in the first line;

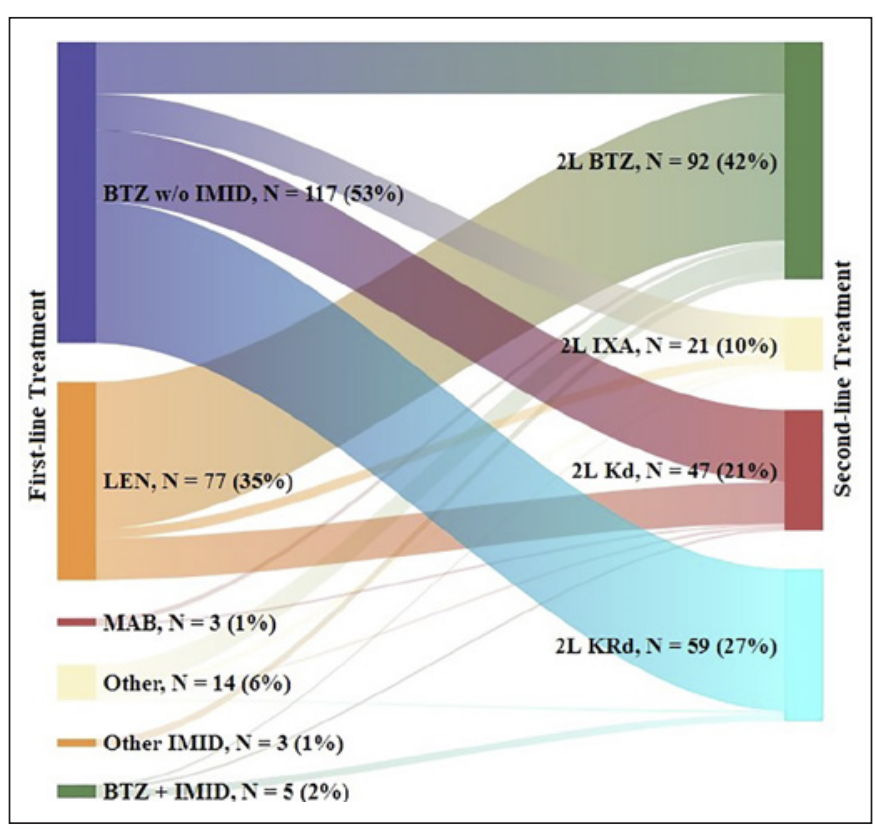

Fig. 2. Treatment shifts from first to second line in patients receiving a PI in second-line treatment $(n=219)$. BTZ, bortezomib; IXA, ixazomib; MAB, monoclonal antibody; w/o, without.

$8.3 \%(n=6)$ had a transplant as second-line therapy and a PI as conventional induction for this SCT. Of the patients receiving a bortezomib-based regimen in the second line, $14.1 \%(n=13)$ had received at least 1 transplant, compared to $49.1 \%(n=52)$ of carfilzomib patients (KRd: $72.9 \%, n=43$; Kd: $19.1 \%, n=9)$ and $33.3 \%(n=7)$ of ixazomib patients (Table 3 ).

\section{Outcomes in Second-Line Therapy}

At the time of documentation, $56.5 \%(n=52)$ of patients receiving bortezomib-based regimens, $77.4 \%(n=82)$ of carfilzomib patients, and $57.1 \%(n=11)$ of ixazomib patients were still undergoing treatment. In the bortezomib group, $82.6 \%(n=76)$ had not yet progressed, compared to $91.5 \%(n=97)$ in the carfilzomib group and $85.7 \%(n=18)$ 


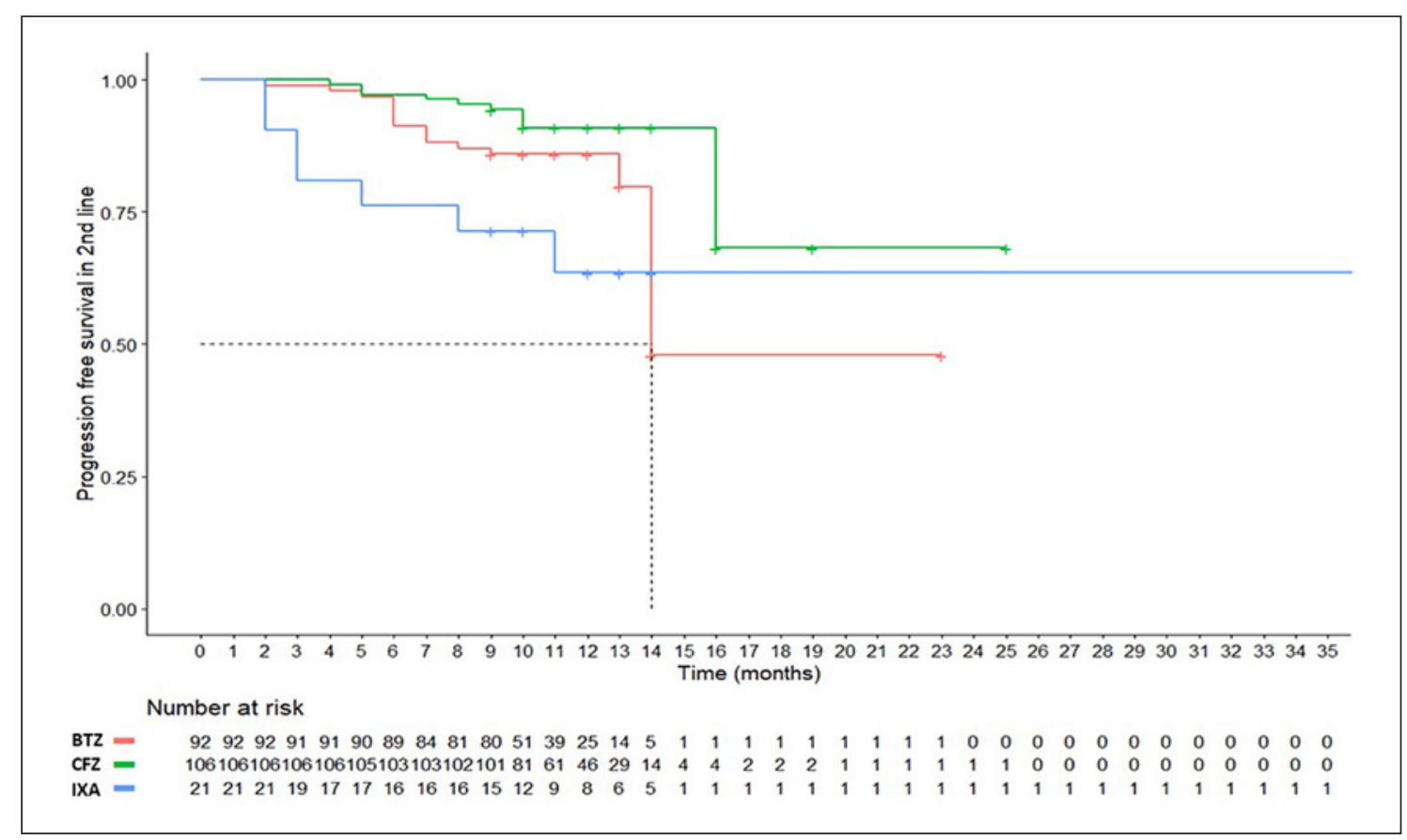

Fig. 3. PFS in second-line treatment for patients who received a PI in second-line treatment. The median was not reached for Kaplan-Meier curves of PFS for patients who received CFZ or IXA in second-line therapy. BTZ, bortezomib; CFZ, carfilzomib-based regimens; IXA, ixazomib.

in the ixazomib group. The median Kaplan-Meier PFS was 14.0 months for bortezomib patients and it was not reached for carfilzomib and ixazomib (Fig. 3). The median KaplanMeier OS was not reached in any group.

Response assessment was performed in $84.5 \%(n=$ 185) of the patients overall. In the bortezomib group, $4.8 \%(n=4)$ achieved a CR, $20.5 \%(n=17)$ achieved a VGPR, and $67.5 \%(n=56)$ achieved a partial response (PR). In the carfilzomib group, $10.1 \%(n=9)$ achieved a CR, $42.7 \%(n=38)$ achieved a VGPR, and $43.8 \%(n=39)$ achieved a PR, with VGPR and CR rates differing between KRd and Kd patients. Only 13 patients receiving ixazomib were assessed for response (Fig. 4).

\section{Patients Receiving a PI in Third-Line Therapy}

Overall, 83 patients had received PI in third-line therapy at the time of data collection. Of these, 12 patients received a bortezomib-based, 51 received a carfilzomibbased, and 20 received an ixazomib-based regimen. Because of the low patient numbers, only top-level results are described here.

\section{Patient Characteristics}

The population receiving PI in third-line therapy was predominantly male $(69.9 \%, n=58)$, with a median age of 66 years (range 40-83) at diagnosis and 70 years (range 41-84) at the initiation of third-line therapy. There were no substantial differences between the PI groups concerning these parameters (online suppl. Table S7).

\section{Disease Characteristics}

At diagnosis, $63.9 \%(n=53)$ had bone pain, $51.8 \%$ $(n=43)$ had anemia, $18.1 \%(n=15)$ had hypercalcemia, and $13.3 \%(n=11)$ had renal dysfunction (online suppl. Table S8). A history of SRE was present in 33.7\% $(n=28)$ of the patients.

\section{Transplant History}

At least 1 transplantation was documented for $48.2 \%$ $(n=40)$ of the patients, mostly in the first line only (online suppl. Table S8).

Treatment Sequences Received in the First through Third Lines

Online supplementary Figure S2A shows a Sankey diagram of treatment shifts from second line to third line; online supplementary Figure S2B shows treatment shifts from first to second to third line in patients receiving a PI in the third line. Online supplementary Table $S 9$ shows an overview of the documented treatment sequences received in the first through third lines of therapy. Patients receiving bortezomib-based regimens in the third line mostly had received a sequence of a PI-based regimen in the first line, an immunomodulatory agent (IMiD)-based regimen in the second line, and the index bortezomibbased regiment in the third line $(75.0 \%, n=9)$. Of the patients receiving a carfilzomib-based regimen in the third line, the most frequent prior sequence $(86.3 \%, n=$ 44) was a PI in the first line, an IMiD in the second line, 


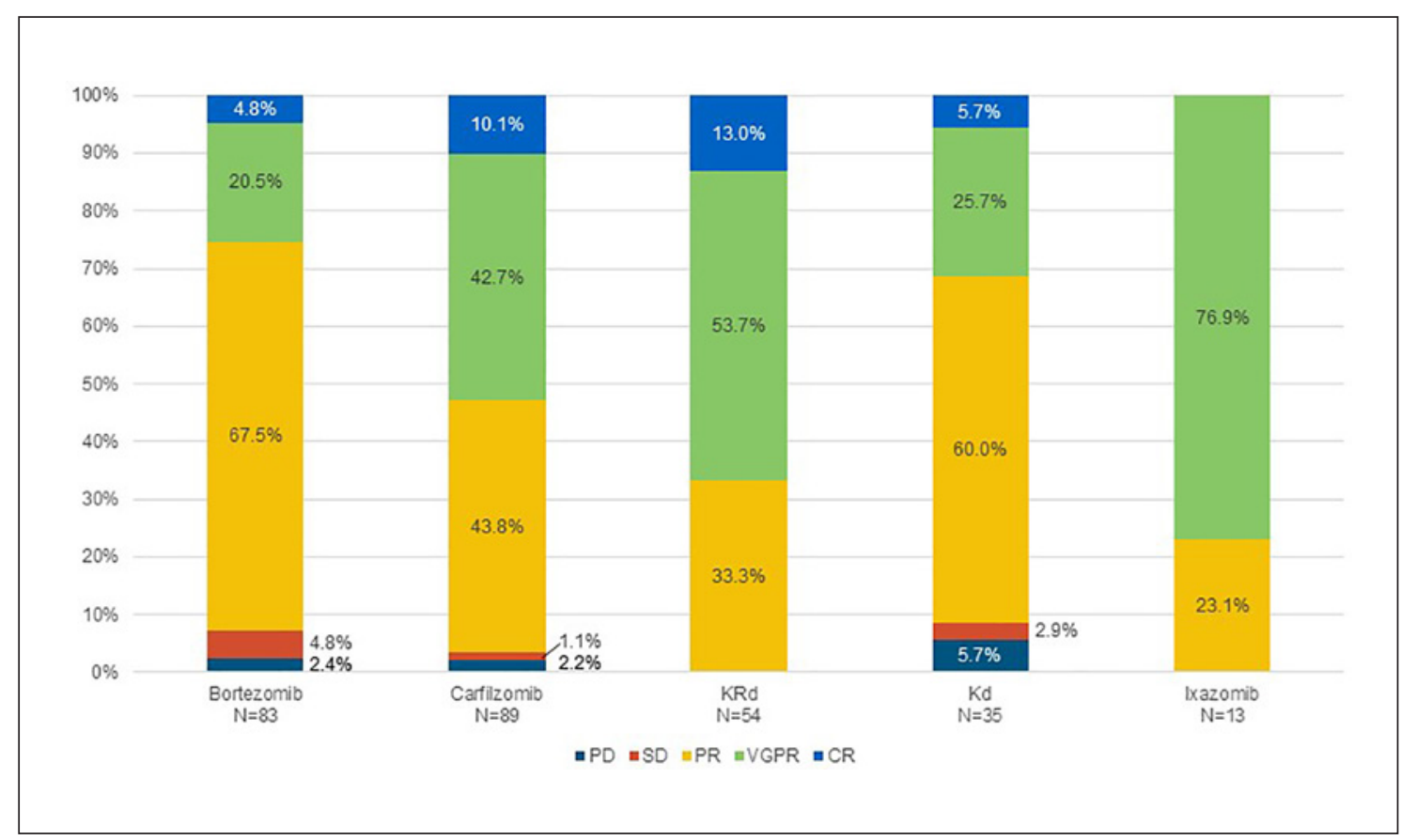

Fig. 4. Best response achieved during second-line PI treatment $(n=219)$. Proportions are based on the number of patients assessed for response $(N)$. PD, progressive disease.

and carfilzomib in third line. Most patients receiving ixazomib in third-line treatment $(55.0 \%, n=11)$ received a triple PI series (online suppl. Table S9). Supportive treatments received in the third line are listed in online supplementary Table S12; of note, 91.6\% $(n=79)$ of the patients received osteoprotective agents.

Outcomes in the First through Third Lines

Online supplementary Table S10 shows an overview of the best responses achieved in first through third treatment lines. These analyses were not stratified by treatment received in the respective prior lines. Due to small sample sizes, results are illustrative. Of the patients receiving bortezomib in third-line therapy, $50.0 \%(n=5)$ achieved a VGPR (no CR were documented). Of the patients receiving carfilzomib in third-line therapy, $2.4 \%$ $(n=1)$ achieved a CR and $38.1 \%(n=16)$ achieved a VGPR. If third-line ixazomib patients, no CR were documented and $64.7 \%(n=11)$ achieved a VGPR (online suppl. Table S10). The time on treatment for patients who received a PI in third-line therapy is shown in online supplementary Figure S3. At the time of this study, the median time on treatment had not been reached for any of the patients during the median follow-up time of 10.0 months. The median PFS and OS had not been reached for any of the third-line patients (data not shown).

\section{Discussion/Conclusion}

This study aimed to describe the demographic and clinical characteristics of patients with MM who had received a PI in second- or third-line treatment at the time of data collection, as well as to describe PI-based treatment patterns and the physician-assessed best response and outcomes in a real-world setting in Germany. The advent of a wide array of new drugs and drug combinations for treating MM, especially in the relapse setting, has made it impossible to conduct head-to-head trials comparing each of these regimens with each other to derive a stringent treatment algorithm for these patients. Additionally, a growing body of research has raised awareness of the relative differences between patients eligible for randomized controlled trials (RCT) and those not eligible [2,17-21], as well as the impact of conditional reimbursement on outcomes [19], triggering a debate on the applicability of pivotal trials to routine clinical practice [22]. Research based on real-world data, e.g., from registries, claims databases, or observational studies of daily practice has thus gained importance and has in some countries become a precondition for reimbursement. This study offers insights from routine clinical care of unselected MM patients receiving PI-based treatments in the second or third line of therapy.

Of the participating physicians, almost all were experienced in using at least 2 different PI in the relapse and/ 
or refractory setting, which suggests that there is informed selection of a given PI for a specific patient and/ or response profile. These identified profiles are discussed below.

In the treatment of MM, strategies are generally tailored along 3 important decision frames, i.e., fitness and transplant eligibility, risk level as determined by ISS stage and cytogenetic risk, and sequencing accounting for refractoriness to prior therapies, especially lenalidomide. Generally, in older patients, a fourth frame exists, i.e., the needs and the individual aims and living conditions, which, however, was not within the scope of this study. In this study, patient characteristics differed by PI type along these decision frames, with younger, fitter, transplant-eligible, lenalidomide-responsive, or IMiD-untreated patients rather receiving triplets based on novel PI. There were no clear patterns regarding risk.

Regarding fitness and transplant eligibility, patients who were younger at diagnosis received carfilzomib- or ixazomib-based regimens while older patients received bortezomib. Within the group of patients who received carfilzomib, KRd patients were younger than Kd patients. This pattern was similar for age at initiation of secondline. Patients still employed or on the job were more likely to receive KRd or ixazomib than Kd or bortezomib. Generally, in Germany patients who are eligible and consent to a transplantation also receive a transplantation [22], which allows more direct interpretation of the profiles of patients receiving or not receiving transplantations without the need to account for logistic, reimbursement, or other obstacles. In the present study, substantially more patients with prior transplantations received KRd in second-line therapy (more than double that of the nextranked ixazomib group). Patients not eligible for transplant were more likely to receive bortezomib or $\mathrm{Kd}$ in second-line therapy. The finding of a preference for bortezomib in transplant-ineligible patients is consistent with a data analysis from the German prospective tumor registry of lymphatic neoplasms (TLN) [2], where $40 \%$ of the patients received bortezomib-melphalan-prednisone (VMP) and 25\% received bortezomib with or without dexamethasone $(\mathrm{V} \pm \mathrm{D})$. However, this analysis of the TLN registry covered the period of 2009-2011, which excludes more recent developments in the treatment landscape. The German MYRIAM registry [21], focusing exclusively on MM and covering all sectors of myeloma treatment in Germany, started in 2017 and thus covers a similar period as the present study. In MYRIAM, patients in second-line therapy who were not eligible for a transplant most frequently received a lenalidomide-based $(70 \%)$ or a carfilzomib-based regimen (39\%), with KRd being the most frequent PI-based regimen (20\%) [21]. However, the authors stated that MYRIAM data were immature at the time of publication of the first interim analysis in 2018.
Regarding the risk level, findings are based on ISS stage at diagnosis. It was not assessed whether they were restaged at initiation of the second line of therapy. Most patients were in advanced stages of disease at diagnosis (ISS II or III), but if they were still in earlier stage (ISS I) KRd or ixazomib was preferred over Kd or bortezomib. In ISS stage I, more patients received KRd, followed by ixazomib as the second most frequent regimen, then bortezomib, and Kd (in descending order). Patients with ISS stage II most often received $\mathrm{Kd}$, followed by KRd, bortezomib, or ixazomib. Patients with ISS stage III equally likely received bortezomib or ixazomib, followed by KRd and $\mathrm{Kd}$. The finding that this real-world population in Germany generally had a higher ISS score is consistent with the CONNECT-MM registry. This registry also showed that real-world populations have a greater proportion of patients with higher ISS stages compared to populations commonly represented in RCT [23], which is of considerable importance when extrapolating protocols tested in RCT to the daily clinical routine. Approximately half of the patients were tested for cytogenetic abnormalities. In all patients tested for cytogenetic aberrations, at least 1 such aberration was detected. Patients receiving KRd were more likely to be tested for cytogenetic aberrations than patients receiving ixazomib, Kd, or bortezomib. The types of aberrations detected varied between the PI groups but followed no clear pattern. A recent review article pointed to the fact that routine collection of cytogenetic abnormalities recognized as conferring a poor prognosis is generally poor and susceptible to technical failure, even in RCT, and the methodology or cut-off to define cytogenetic risk categories is inconsistent with definitions of clone sizes for del/17p for instance ranging from abnormalities in a single cell, through 1.5$7.5 \%$ of cells, up to $60 \%$ of cells [22].

Regarding treatment sequencing, patients receiving bortezomib in second-line treatment were most likely to have received prior lenalidomide. Those receiving a carfilzomib or ixazomib were most likely to have received prior bortezomib. In the group of patients receiving carfilzomib, there was a difference between KRd and Kd patients where KRd patients were not pretreated with lenalidomide, whereas approximately one third of the $\mathrm{Kd}$ patients were lenalidomide pretreated. These patterns evolved from an earlier study conducted in 2014 in 6 Western European countries including Germany, where most patients had either received bortezomib or thalidomide in first-line treatment with no documented frontline lenalidomide $[13,14]$. There is to date no clear guidance on sequencing, as treatment choices are extremely complex. Factors such as individual characteristics and their representation or nonrepresentation in clinical trials, clonal heterogeneity in the context of response to treatment, and multiple other factors need to be under- 
stood. International guidelines thus vary in their recommendations and their translation into clinical practice across Europe is inconsistent, as a recent literature review has shown [16].

There were pronounced differences between groups regarding the best response achieved, noting that response assessments were not done according to the criteria issued by the IMWG but rather were done according to the physicians' usual practice. Ixazomib results need to be interpreted with caution due to the low number of patients receiving this PI in the present study and are thus not discussed in the context of the existing literature. The CR rates were highest in patients receiving $\mathrm{KRd}(13.0 \%)$, followed by $\mathrm{Kd}$ (5.7\%) and bortezomib (4.8\%). The VGPR rates were highest in patients receiving ixazomib (76.9\%), followed by KRd (53.7\%), Kd (25.7\%), and bortezomib (20.5\%). All patients with KRd and ixazomib were assessed as achieving at least a PR. In the ENDEAVOR trial, CR or better was achieved by $13 \%$ of $\mathrm{Kd}$ and $6 \%$ of Vd patients; VGPR were reached by 54 versus $29 \%$ of patients, and PR were achieved by 22 versus $34 \%$ of patients [24]. In the ASPIRE trial, $31.8 \%$ of patients receiving $\mathrm{Kd}$ achieved a CR or better, and $69.9 \%$ achieved a VGPR or better [25].

Bortezomib patients reached a median PFS (14.0 months); for all other treatment groups, PFS was not reached during the time of observation. Patients receiving Vd in the ENDEAVOR trial [24] reached a median PFS of 9.4 months (95\% CI 8.4-10.4) over the 30-month trial duration, compared to 18.7 months (95\% CI 15.6-NE) in patients receiving $\mathrm{Kd}$. In patients receiving $\mathrm{KRd}$ in the ASPIRE trial, the median PFS over the 48-month trial duration was 26.3 months [25]. As already pointed out, there is a growing body of evidence that patient characteristics differ between this study of routine clinical practice and pivotal RCT [17-20]. An analysis of the representativeness of the RCT ASPIRE, TOURMALINE-MM1, POLLUX, and ELOQUENT-2 in real-world relapsed and/or refractory MM patients showed that the 3-year overall survival was longer for patients classified as RCT eligible compared to RCT ineligible patients [26].

Regarding comorbidities and their potential impact on the choice of treatment, the distribution of patients with a history of SRE was similar among the groups, except for ixazomib patients who were more likely to have had an SRE history. Patients with renal impairment were more likely to receive bortezomib or ixazomib than carfilzomib-based regimens. Interestingly, there was no observable difference regarding renal function between patients receiving $\mathrm{KRd}$ and $\mathrm{Kd}$, despite necessary precautions in patients with renal impairment who are planned to receive lenalidomide [27]. Additionally, an equal proportion of patients with a history of neuropathy received bortezomib compared to carfilzomib, despite a known causal relationship between bortezomib use and periph- eral neuropathy [28, 29]. Ixazomib patients were less likely to have had a history of neuropathy in the present study than patients in the other groups. Patients with a history of cardiovascular disease were less likely to have been administered a carfilzomib-based regimen, although there was a difference between $\mathrm{KRd}$ and $\mathrm{Kd}$.

The time between diagnosis and the initiation of second-line therapy and the time between the first- and second-line therapies were assessed. For both periods, patients receiving carfilzomib, especially KRd, had a pronouncedly longer time between diagnosis and the initiation of second-line therapy. The period between the end of the first-line therapy and the initiation of the second-line therapy was substantially longer in transplanted compared to nontransplanted patients.

The following top-level observations were made in patients receiving a PI in third-line therapy; the discussion is kept to a minimum due to low patient numbers. The median time from diagnosis to initiation was shorter in carfilzomib patients than in bortezomib or ixazomib patients. More patients with ISS stage III received bortezomib or carfilzomib than ixazomib. Interestingly, in terms of work status the opposite was true for patients receiving a PI in second-line therapy, with more bortezomib patients still working or being employed than those receiving novel PI (carfilzomib and ixazomib). Regarding treatments received, almost all of the patients had received a sequence of a bortezomib-based regimen without an IMiD in first-line therapy, followed by an IMiD in second-line therapy and a PI in third-line therapy.

This study has some limitations. Firstly, no comparison of groups can be made due to the study design and the purely descriptive statistics. The fact that this study was set up to investigate separate groups of patients who had received a PI in second- or third-line treatment and their respective characteristics and prior treatments precludes interpretations regarding patient flow through the treatment lines. Special precautions need to be taken when interpreting response data and Kaplan-Meier plots, as they were only an exploratory endpoint and most patients were still on treatment when the data were extracted from the electronic medical records.

Importantly, this study was done in 2017 and the treatment landscape has evolved since then. For instance, lenalidomide in first-line therapy was only just approved at the time of study. Additionally, the quota for ixazomib was lower than those for other PI due to the launch of ixazomib only in January 2017, rendering findings for lenalidomide and ixazomib unrepresentative of a more mature clinical landscape. Moreover, the number of patients receiving ixazomib was rather small $(\sim 20$ in each treatment line), mandating caution in data interpretation. Also, some off-label use was observed for ixazomib and bortezomib due to the design of the CRF, which allowed 
observation of any treatment combination administered in clinical practice - except for carfilzomib. In first-line treatment, all prescriptions could have been within clinical trials ( $n=5$ patients, i.e., 4 carfilzomib and 1 ixazomib) or within a compassionate use program. In the relapse setting, clinical trial participants were excluded.

Another limitation was that only 44 physicians from 44 different sites could be recruited, compared to the 80 initially planned. While the study collected a robust number of patient records (302, compared with the 320 originally planned), they only represent the management patterns of 44 different sites and are thus less geographically representative than the 80 sites that were initially planned. However, the regional and hospital type distributions were representative, minimizing this bias.

Most of the physicians (41 out of 44) had initiated patients on $>1$ PI during the documentation period. The complete anonymization of the data collected allows no link between patients and their physicians' setting (office based or hospital based, whether working in a transplant center or not) or region. Thus, treatment preferences for different settings cannot be discerned.

Overall the retrospective nature of the study design and the patient information itself limit the outcomes and interpretation. Certain variables could not be assessed, e.g., distance to care for patients and patient preferences, which could have an impact on treatment decisions (e.g., oral vs. i.v. application).

\section{Conclusions}

This study shows clear patient profiles for each type of PI observed. In second-line treatment, younger, fitter, transplant-eligible patients rather received triplets based on novel PI such as KRd or IRd. Patients treated with lenalidomide in first-line treatment mostly received lenalidomide-sparing regimens in second-line treatment. In high-risk patients no clear treatment patterns could be ascertained due to the limited sample size. This study clearly shows that research based on real-world data is important to fill the evidence gap that comes from the improbability of conducting mutual head-to-head studies in a condition with multiple possible treatment options. The results also show the importance of generating real-world data to understand management practices and define areas of improvement in routine clinical practice.

\section{Statement of Ethics}

This study protocol was submitted to the Ethics Committee of Nord-Rhein (Cologne) on December 13, 2017. Upon review, the committee determined that no approval was necessary for this study due to the retrospective and anonymous nature of data collection. Patient informed consent was not necessary, as confirmed by the committee.

\section{Conflict of Interest Statement}

H. Tilman Steinmetz has received consultancy fees from Accord Healthcare, Amgen, Ariad, BMS, Boehringer, Celgene, HexalSandoz, Novartis, Janssen-Cilag, Omnicare, Oncopeptides, Otsuka, Pfizer, Sanofi, Shire, TAD, and Vifor, as well as fees as principal investigator in investigator-initiated trials, noninterventional studies, or registries from Accord Healthcare, Amgen, Celgene, Novartis, and Vifor, and travel fees from Alexion, Amgen, Bayer, BMS, Boehringer, Celgene, Janssen-Cilag, Novartis, Omnicare, and Sanofi. Andrea Lebioda, Moushmi Singh, Sebastian GonzalezMcQuire, Achim Rieth, and Martina Schoehl are employees of Amgen. Andrea Lebioda, Sebastian Gonzalez-McQuire, Achim Rieth, and Moushmi Singh hold Amgen stock. Wolfram Poenisch has received consultancy fees from Amgen, Celgene, Janssen, Takeda, and research funding from Amgen, Celgene, Janssen, and Mundipharma.

\section{Funding Sources}

This study was sponsored by Amgen GmbH, Munich, Germany. Study conduction and statistical analysis were performed by Kantar Health, Paris, France, sponsored by Amgen Ltd., Uxbridge, UK.

\section{Author Contributions}

H. Tilman Steinmetz, Andrea Lebioda, Moushmi Singh, Sebastian Gonzalez-McQuire, Achim Rieth, Martina Schoehl, and Wolfram Poenisch participated in content planning sessions for this paper, interpreted the data, reviewed and commented on drafts, and approved the final version of this paper for publication. All of the authors had full insight into all of the statistical analyses and were involved in drafting and reviewing of this paper.

\section{Data availability}

Amgen holds the source data and all authors had full access to the data. Qualified researchers may request data from Amgen clinical studies. Complete details are available at the following: http:// www.amgen.com/datasharing.

\section{Acknowledgement}

Margit Hemetsberger of Hemetsberger Medical Services, Vienna, Austria, helped with writing and editing of this paper and was sponsored by Amgen Ltd., Uxbridge, UK. 


\section{References}

1 Raab MS, Podar K, Breitkreutz I, Richardson PG, Anderson KC. Multiple myeloma. Lancet. 2009 Jul;374(9686):324-39.

2 Knauf W, Abenhardt W, Aldaoud A, Nusch A, Grugel R, Münz M, et al.; TLN Study Group. Treatment of Non-transplant patients with multiple myeloma: routine treatment by office-based haematologists in Germanydata from the prospective Tumour Registry Lymphatic Neoplasms (TLN). Oncol Res Treat. 2014;37(11):635-6.

3 Koch-Institut R. Krebs in Deutschland für 2013/2014. Robert Koch-Institut; 2017.

4 Surveillance, Epidemiology, and End Results Program. Cancer stat facts: myeloma. Bethesda: SEER; 2017.

5 Ferlay J, Steliarova-Foucher E, Lortet-Tieulent J, Rosso S, Coebergh JW, Comber H, et al. Cancer incidence and mortality patterns in Europe: estimates for 40 countries in 2012. Eur J Cancer. 2013 Apr;49(6):1374-403.

6 Kaatsch P, Spix C, Katalinic A, et al. Krebs in Deutschland für 2013/2014. Berlin: Robert Koch-Institut (Hrsg); 2017, vol 11.

7 Wörmann B, Driessen C, Einsele H, et al. Onkopedia Leitlinien Multiples Myelom. Berlin: Springer; 2018.

8 Moreau P, San Miguel J, Sonneveld P, Mateos MV, Zamagni E, Avet-Loiseau H, et al. Multiple myeloma: ESMO Clinical Practice Guidelines for diagnosis, treatment and follow-up. Ann Oncol. 2017;28:iv52-61.

9 Rajkumar SV, Dimopoulos MA, Palumbo A, Blade J, Merlini G, Mateos MV, et al. International Myeloma Working Group updated criteria for the diagnosis of multiple myeloma. Lancet Oncol. 2014 Nov;15(12):e538-48.

10 Cavo M, Rajkumar SV, Palumbo A, Moreau $\mathrm{P}$, Orlowski R, Bladé J, et al.; International Myeloma Working Group. International Myeloma Working Group consensus approach to the treatment of multiple myeloma patients who are candidates for autologous stem cell transplantation. Blood. 2011 Jun; 117(23): 6063-73.

11 Avet-Loiseau H, Facon T. Front-line therapies for elderly patients with transplant-ineligible multiple myeloma and high-risk cytogenetics in the era of novel agents. Leukemia. 2018 Jun;32(6):1267-76.

12 Mohty M, Cavo M, Fink L, Gonzalez-McQuire S, Leleu H, Mateos MV, et al. Understanding mortality in multiple myeloma: findings of a European retrospective chart review. Eur J Haematol. 2019 Aug;103(2):10715.

13 Yong K, Delforge M, Driessen C, Fink L, Flinois A, Gonzalez-McQuire S, et al. Multiple myeloma: patient outcomes in real-world practice. Br J Haematol. 2016 Oct;175(2): 252-64.

14 Raab MS, Cavo M, Delforge M, Driessen C, Fink L, Flinois A, et al. Multiple myeloma: practice patterns across Europe. Br J Haematol. 2016 Oct;175(1):66-76.

15 Coriu D, Dytfeld D, Niepel D, Spicka I, Markuljak I, Mihaylov G, et al. Real-world multiple myeloma management practice patterns and outcomes in selected Central and Eastern European countries. Pol Arch Intern Med. 2018 Sep;128(9):500-11.

16 Cavo M, Terpos E, Bargay J, Einsele H, Cavet J, Greil R, et al. The multiple myeloma treatment landscape: international guideline recommendations and clinical practice in Europe. Expert Rev Hematol. 2018 Mar;11(3): 219-37.

17 Costa LJ, Hari PN, Kumar SK. Differences between unselected patients and participants in multiple myeloma clinical trials in US: a threat to external validity. Leuk Lymphoma. 2016 Dec;57(12):2827-32.

18 Klausen TW, Gregersen H, Abildgaard N, Andersen NF, Frølund UC, Gimsing P, et al. The majority of newly diagnosed myeloma patients do not fulfill the inclusion criteria in clinical phase III trials. Leukemia. 2019 Feb; 33(2):546-9.

19 Gaultney JG, Franken MG, Uyl-de Groot CA, et al. Experience with outcomes research into the real-world effectiveness of novel therapies in Dutch daily practice from the context of conditional reimbursement. Health Policy. 2015 Feb;119(2):186-94.

20 Knauf W, Aldaoud A, Hutzschenreuter U, Klausmann M, Dille S, Wetzel N, et al.; and the TLN-Group (Tumour Registry Lymphatic Neoplasms). Survival of non-transplant patients with multiple myeloma in routine care differs from that in clinical trials-data from the prospective German Tumour Registry Lymphatic Neoplasms. Ann Hematol. 2018 Dec;97(12):2437-45

21 Knauf W, Engelhardt M, Lipke J, et al. MYRIAM: Prospective, intersectoral Real World cohort study for treatment and outcome of myeloma patients in Germany. Oncol Res Treat. 2018;41(190):574.

22 Richardson PG, San Miguel JF, Moreau P, Hajek R, Dimopoulos MA, Laubach JP, et al. Interpreting clinical trial data in multiple myeloma: translating findings to the realworld setting. Blood Cancer J. 2018 Nov; 8(11):109.

23 Shah JJ, Abonour R, Gasparetto C, Hardin JW, Toomey K, Narang M, et al. Analysis of Common Eligibility Criteria of Randomized Controlled Trials in Newly Diagnosed Multiple Myeloma Patients and Extrapolating Outcomes. Clin Lymphoma Myeloma Leuk. 2017 Sep;17(9):575-583.e2.

24 Dimopoulos MA, Moreau P, Palumbo A, Joshua D, Pour L, Hájek R, et al.; ENDEAVOR Investigators. Carfilzomib and dexamethasone versus bortezomib and dexamethasone for patients with relapsed or refractory multiple myeloma (ENDEAVOR): a randomised, phase 3, open-label, multicentre study. Lancet Oncol. 2016 Jan;17(1):27-38.

25 Stewart AK, Rajkumar SV, Dimopoulos MA, Masszi T, Špička I, Oriol A, et al.; ASPIRE Investigators. Carfilzomib, lenalidomide, and dexamethasone for relapsed multiple myeloma. N Engl J Med. 2015 Jan;372(2):142-52.

26 Chari A, Romanus D, Farrelly E, et al. Randomized clinical trial (RCT) representativeness \& outcomes in relapsed/refractory multiple myeloma (RRMM) real world patients: comparison of ASPIRE, TOURMALINEMM1, POLLUX, \& ELOQUENT RCTS 23rd European Association of Haematology Congress. Stockholm; 2018.

27 Ludwig H, Delforge M, Facon T, Einsele H, Gay F, Moreau P, et al. Prevention and management of adverse events of novel agents in multiple myeloma: a consensus of the European Myeloma Network. Leukemia. 2018 Jul; 32(7):1542-60.

28 Delforge M, Bladé J, Dimopoulos MA, Facon T, Kropff M, Ludwig H, et al. Treatment-related peripheral neuropathy in multiple myeloma: the challenge continues. Lancet Oncol. 2010 Nov;11(11):1086-95.

29 Arastu-Kapur S, Anderl JL, Kraus M, Parlati F, Shenk KD, Lee SJ, et al. Nonproteasomal targets of the proteasome inhibitors bortezomib and carfilzomib: a link to clinical adverse events. Clin Cancer Res. 2011 May;17(9): 2734-43. 